\title{
IS THE PULSAR EMISSION PRODUCED BY SUPERLUMINALLY MOVING CHARGED PATTERNS?
}

\author{
Housang Ardavan \\ Institute of Astronomy, University of Cambridge
}

\begin{abstract}
There are no physical principles disallowing the existence of extended sources of the electromagnetic field which move faster than light in empty space (Bolotovskii and Ginzburg 1972). Chargecurrent distributions whose patterns rotate around a fixed axis rigidly, and so have a phase speed that is greater than the speed of light in vacuo outside the light cylinder, emit radiation-different from both Cherenkov and synchrotron radiation-which has the following characteristics:
\end{abstract}

1. It arises almost exclusively from the vicinity of those points within the source which, at the emission time, approach the observer with the speed of light and with zero acceleration.

2. It consists of the superposition of a (continuous) set of narrow radiation beams whose flux densities fall off with the radial distance $R$ from the source like $R^{-1}$ (rather than $R^{-2}$ ), and so it is intrinsically coherent.

3. It has a power-law spectrum with no exponential cut-off, at high harmonics of the rotation frequency, whose index lies between -1 and -3 if the longitudinal distribution of the source density is pulse-like.

4. It consists of two concurrent elliptically polarized signals whose position angles are predominantly orthogonal and simultaneously vary in direction in the course of each rotation.

I suggest that this radiation, here referred to as Schott radiation (cf. Schott 1912), may in fact be that received from pulsars.

\section{Introduction}

The average waveforms from pulsars, when observed in an inertial frame marked by the cylindrical polar coordinates $\left(r_{\mathrm{P}}, \varphi_{\mathrm{P}}, z_{\mathrm{P}}\right)$ whose $z_{\mathrm{P}}$-axis is coincident with the rotation axis of the central neutron star, vary both temporally $\left(t_{P}\right)$ and azimuthally $\left(\varphi_{\mathrm{P}}\right)$, as functions of the single variable $\varphi_{P}-\omega t_{P}$, with an angular frequency $\omega$ which is remarkably constant. Since a rigidly rotating radiation pattern can only arise from a rigidly rotating source distribution, this implies that the electric charges and currents, in the magnetosphere of the neutron star, which produce the observed radiation must have densities that also depend on the source coordinates $\varphi$ and $t$ in the combination $\varphi-\omega t$ only, and that $\omega$ is a constant. [Here $\left(r_{\mathrm{P}}, \varphi_{\mathrm{P}}, z_{\mathrm{P}}, t_{\mathrm{P}}\right)$ and $(r, \varphi, z, t)$ denote the space-time coordinates of the observation point and the source points, respectively.] Thus the magnetospheric plasma distribution must have a rigidly rotating pattern (see, e.g., Mestel 1971, Ardavan 1981, and da Costa and Kahn 1985); though, of course, none of the charged particles which constitute the source, and provide the medium for the propagation of the pattern associated with the source, are themselves constrained to be co-rotating.

The fact that the pattern's speed $r \omega$ exceeds $c$, the speed of light in vacuo, outside the light cylin. der $r=c / \omega$ does not violate the requirements of special relativity. The superluminally moving pattern is created by the coordinated motion of aggregates of subluminally moving particles. To give an example, the charge separation caused by the collective motion of a set of particles would create a wave packet of charge (a plasmon) which, because it has no inertia, can propagate with a superluminal phase velocity. The distinction between a source which consists of a moving material object and a source which represents a moving pattern, however, is not in any way reflected in the field equations, so that the electromagnetic field which arises from such a wave packet is precisely the same as that which would arise from a similarly moving massive charged object. Nor does the source have to be specifically a moving packet of charge for this to be true; any macroscopic charge-current, the changes in whose distribution pattern propagate faster than light, will, as a source of the electromagnetic field, act as though it were produced by the faster-thanlight motion of actual charged particles. A full discussion of this point is given in Bolotovskii and Ginzburg (1972). 


\section{Characteristics of Schott radi- ation}

The near-zone properties of the electromagnetic field produced by a charge-current distribution for which the charge density $\rho$ and the cylindrical components of the current density $\mathbf{j}$ depend on the time $t$ and the azimuthal angle $\varphi$ in the combination $\varphi-\omega t$ only, have already been analyzed in Ardavan (1989). Here I describe certain far-field properties of this radiation in the observable part of the spectrum, i.e., for the frequencies $m \omega$ which correspond to high harmonics $\left(m>10^{8}\right)$ of the rotation frequency $\omega$. The mathematical derivation of these properties will be presented elsewhere (see also Ardavan 1991b).

The radiation field of any extended source can be built up by the superposition of the radiation fields of the volume elements which constitute it, i.e., it can be obtained from an associated Green's function for the problem. The point source whose potential acts as the Green's function for the present problem is simply a point charge which moves on a circle of radius $r$ with the constant angulay frequency $\omega$. The Lienard-Wiechert potentials for such a source are well known from the study of synchrotron radiation; when $r>c / \omega$ and the source moves superluminally, these potentials become singular on a surface which I shall refer to as the caustic.

The caustic surface, whose cross section with the plane of the orbit is shown in figure 1, constitutes the envelope of the spherical field wavelets emanating from the source point. It begins issuing from the source in the form of a cone with the same opening angle as that of the Cherenkov shock and, after joining a second sheet, eventually develops into a tube-like surface which spirals around the rotation axis. The two sheets of the caustic meet along a U-shaped cusp curve on which the rays associated with the field wavelets further focus and so the potentials have higher-order singularities. The closer the position of the source point to the light cylinder, the smaller is the cross-sectional area of the caustic. For $r=c / \omega$, the volume enclosed by the caustic shrinks to zero and the whole surface collapses onto the cusp curve, which in this limit lies in the plane of the orbit. ${ }^{1}$

The fields at the caustic are infinitely strong for a source which is point-like, but in the case

\footnotetext{
${ }^{1}$ For a detailed mathematical account of this caustic see Ardavan (1989); the caustic in question is also encountered in the context of the impulsive noise produced by supersonic helicopter rotors, an acoustic radiation which has many features in common with the electromagnetic radiation received from pulsars, see, e.g., Lowson and Jupe (1974), Schmitz and Yu (1986), and Ardavan (1991a).
}

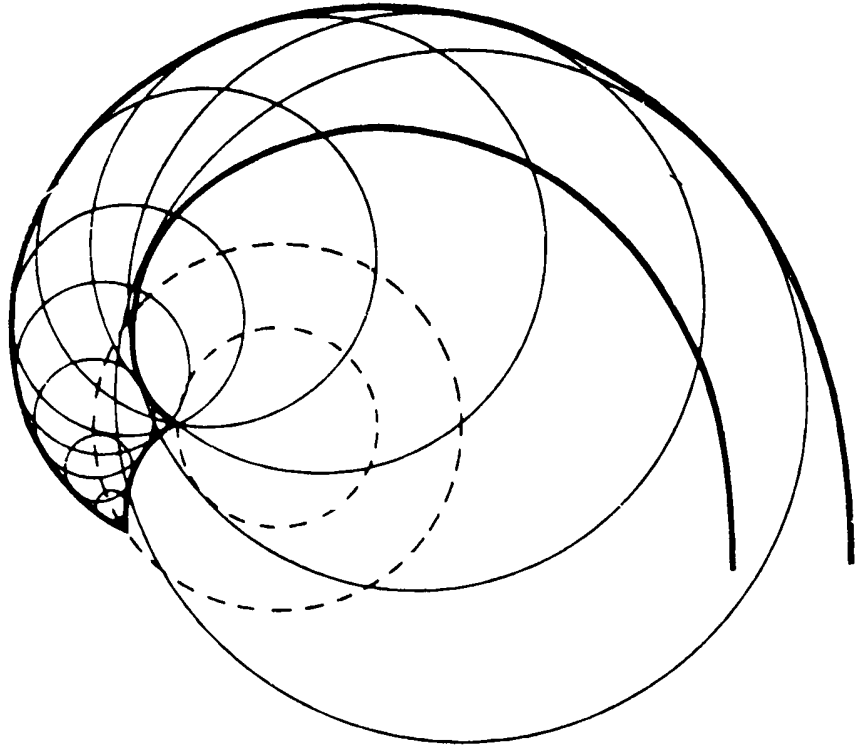

Figure 1 The envelope of the spherical field wavelets emanating from a superluminally moving source point in circular motion. The heavier curves show the cross section of the envelope with the plane of the orbit of the source. The larger of the two broken circles designates the orbit and the smaller the light cylinder.

of an extended localized source which has a nonsingular density, they are finite and they can be observed over a wide solid angle. An analysis based on the method of stationary phase shows that the high frequency emission received at the point $\left(r_{\mathbf{P}}, \varphi_{\mathbf{P}}, z_{\mathrm{P}}\right)$ from a superluminally moving extended source arises almost exclusively from the neighborhood of those points within the source which, at the (retarded) emission time $t$, instantaneously approach this observation point with the speed of light and zero acceleration. The electromagnetic waves originating from all other points of the source interfere destructively and so make a relatively minor contribution towards the observed radiation. The set of coherently emitting source points have a fixed (one-dimensional) locus relative to the observation point, so that the variation in the intensity of the observed radiation during a rotation period essentially reflects the longitudinal distribution of the charge-current density in the rotating frame.

Because the field wavelets emanating from the coherently emitting source elements crowd together in the direction of the observation point to such an extent that the ratio of the observation to the emission time intervals is almost zero, the amplitude of the electromagnetic radiation that arises from the constructive interference of these wavelets has to be correspondingly large if the flux of energy is to remain constant. In fact, from an asymptotic analysis of the problem it follows that the flux density of the present radiation falls off with the distance $R$ away from the source like $R^{-1}$ rather than $R^{-2}$. The total 
flux of energy across a sphere of radius $R$ centered at the source is of course the same for all values of $R$; however, the electromagnetic waves observable in the vicinity of the caustic have amplitudes which decrease only like $R^{-1}$. This means that the brightness temperature associated with such a radiation exceeds that of an equivalent spherically decaying emission by a large factor ( $>10^{18}$ for a source which is at a distance of $1 \mathrm{kpc}$ ), and that Schott radiation is hence an intrinsically coherent emission. In addition, the associated narrowing with distance of the widths of the individual radiation beams-arising from the (one-dimensional) loci of the coherently emitting source points-implies that the signal that is received during each rotation period consists of the superposition of a (continuous) set of extremely narrow 'spikes' (micro- and nano-pulses).

The spectrum of the radiation is determined not only by the variations of the source densities $(\rho, \mathbf{j})$ as functions of $\varphi-\omega t$, but in addition by the Fourier transform of the Green's function that dictates the radiation efficiency of each source element. If the longitudinal distribution of the electric current density is in the form of a sharp pulse, so that the spectral distribution of the source density contains all the frequencies, then the power emitted into the $m^{\text {th }}$ harmonic of the rotation frequency varies like $m^{-1}$ with the harmonic number $m \gg 1$. On the other hand, if the electric current density has a step-function discontinuity, then the radiation has a power-law spectrum with the index -3 . Since the emission involves caustics, at which the wave fronts crowd together to such an extent that the wavelength of the radiation is Doppler-shifted to zero, its spectrum does not have the exponential cut-off which appears in the synchrotron spectrum and so in general extends over a wide range of frequencies (from radio waves to $\gamma$ rays) with a power-law decay.

Th: orientation of the electric field vector of the radiat ${ }_{1}$ on at the observation point is determined by the orientation of the poloidal current density at the locus of the points from which the constructively interfering waves originate. Thus, when arising from a macroscopic current distribution whose density, $j$, retains a well-defined direction along the filarnentary locus of the coherently emitting source points, Schot t radiation is elliptically polarized. Moreover, the change (required by charge conservation) in the direction of the vector $\mathbf{j}$ around the circular trajectory of each source point is mapped, in the course of a rotation period, into a corresponding continuous change in the orientation of the observed electric field vector.

The stronger fields on the caustics arise from the constructive interference of two waves which, though emitted at different retarded times, are re- ceived simultaneously. The coherent radiation that is observed at any given instant, therefore, is composed of two modes differing in certain characteristics. For most relevant topologies of the vector field $\mathbf{j}$, the polarization position angles of these two concurrent modes happen to be almost orthogonal.

\section{Concluding remarks}

Because the sources of the emission described here have a phase speed which exceeds the speed of light in vacuo rather than a bulk speed exceeding the speed of light in a medium, Schott radiation is fundamentally different from the synchrotronCherenkov radiation that is discussed by Schwinger et al. (1976) and other authors (see, e.g., Bonin et al. 1986). The refractive effects of the medium through which the radiation propagates come into play only when the charged particles which form the medium are not included in the sources of the radiation. In the canonical oblique-rotator model of pulsars, where the entire plasma present outside the light cylinder has a rotating distribution pattern and so acts as a source of Schott radiation, there is in fact no medium to be considered.

Of course here, as in all other discussions of the oblique-rotator model, the question as to how the magnetic field of the neutron star affects a coupling between the star's rotation and that of the distribution pattern of its magnetospheric plasma is left unanswered. It is remarkable, however, that despite this missing link in the chain of the argument, the emission produced by a mechanism so basic as that described here should be in all its salient features the same as the emission that is received from pulsars.

It should be emphasized that none of the earlier arguments against the light-cylinder models of pulsars apply to the mechanism described here. The energetic requirements of creating a charged wave pattern which propagates superluminally are minimal compared to those of producing a massive plasma which moves relativistically. Moreover, the existing scintillation observations of pulsars can only specify the extent of the emitting region transverse to the line of sight. The conclusions normally drawn from these data concerning the altitude of the emitting region above the neutron star, which are themselves dependent on the adoption of an emission mechanism in which the radiation is beamed along the open lines of force of a dipolar magnetic field, are not in fact valid in the context of the present mechanism.

Acknouledgments: I thank F. D. Kahn and V. S. Beskin for stimulating and helpful conversations. 